\title{
The Effectiveness of Face Detection Algorithms in Unconstrained Crowd Scenes
}

\author{
Jeremiah R. Barr, Kevin W. Bowyer, Patrick J. Flynn \\ Department of Computer Science and Engineering \\ University of Notre Dame \\ Notre Dame, IN 46656 \\ jbarr1,kwb, flynnend.edu
}

\begin{abstract}
The 2013 Boston Marathon bombing represents a case where automatic facial biometrics tools could have proven invaluable to law enforcement officials, yet the lack of robustness of current tools in unstructured environments limited their utility. In this work, we focus on complications that confound face detection algorithms. We first present a simple multi-pose generalization of the Viola-Jones algorithm. Our results on the Face Detection Data set and Benchmark (FDDB) show that it makes a significant improvement over the state of the art for published algorithms. Conversely, our experiments demonstrate that the improvements attained by accommodating multiple poses can be negligible compared to the gains yielded by normalizing scores and using the most appropriate classifier for uncontrolled data. We conclude with a qualitative evaluation of the proposed algorithm on publicly available images of the Boston Marathon crowds. Although the results of our evaluations are encouraging, they confirm that there is still room for improvement in terms of robustness to out-of-plane rotation, blur and occlusion.
\end{abstract}

\section{Introduction}

Two explosions killed or injured many bystanders at the 2013 Boston Marathon [7]. Thousands of individuals photographed or recorded the events before the bombing, the incident and its aftermath, while surveillance cameras collected hours of footage. Ideally, automated face recognition tools could have aided law enforcement officials in processing this mass of data and identifying the suspects.

Unfortunately, the crowded scenes were too challenging for existing facial biometrics methods and databases [4]. Klontz and Jain [16] conducted a case study on the performance of commercial face matchers in identifying the bombing suspects. The results showed that the algorithms tended to rank images of the suspects below the images of tens, hundreds or thousands of other people in terms of their

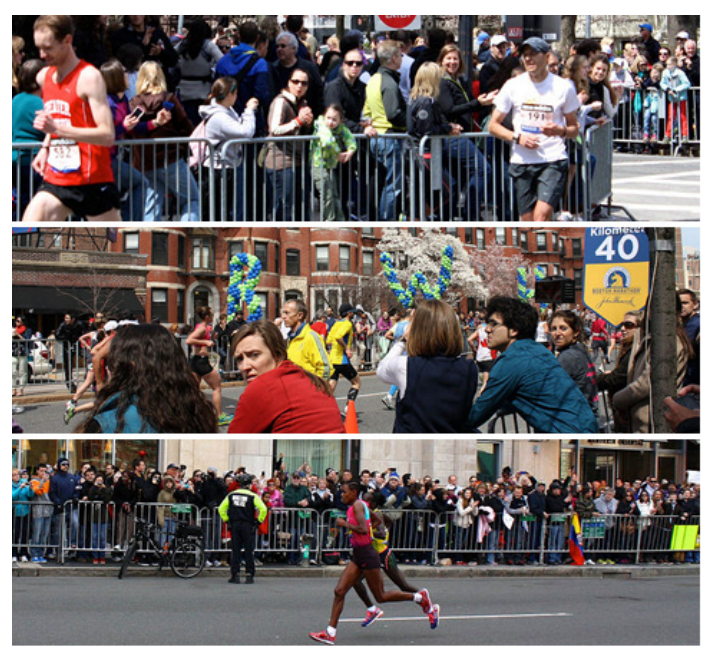

Figure 1. Examples of images taken at the Boston Marathon. These images are attributable to S. Su [25] and available under the Creative Commons Attribution 2.0 Generic license [2].

match strengths.

Face recognition pipelines must detect faces in an image or video prior to performing identification [18, 31, 32]. However, the limitations of current detectors made it difficult to isolate faces, much less identify the suspects. This is because face detection in unstructured crowd scenes is still largely an open problem.

Figure 1 illustrates some of the difficulties inherent to the Boston Marathon imagery. Crowd members often faced away from the cameras recording the event. Some of the faces were out of focus or located far from the cameras. People occupying positions near the cameras often occluded individuals located behind them. The effects of out-of-plane rotation, blur and occlusion confound detectors by increasing the intra-class variance of faces.

Here, we analyze the impact of these factors on a strong performing multi-pose generalization of the Viola-Jones Haar-like feature cascade [27], both on the popular Face 
Detection Data set and Benchmark (FDDB) [11] and images of the Boston Marathon. Our contributions are three fold:

1. We introduce a multi-pose algorithm that employs simple score normalization and fusion techniques to combine information from independent pose-specific detectors. We show results obtained on the FDDB data set, which contains a large number of photographs of crowds. Our algorithm surpasses the state of the art for published methods on this benchmark using relatively simple techniques.

2. We present analyses of the effects of blur and occlusion on the proposed algorithm. Although pose is still a major challenge in face detection, the results suggest that blur and occlusion need more attention from the face detection research community.

3. We provide a case study that highlights the ability of the proposed method to find faces in images of the Boston Marathon. The results illustrate the limitations of current face detectors, especially with respect to their abilities to cope with simultaneous out-of-plane rotation, blur and occlusions.

In the sections below, we review the related literature (Section 2) and describe the design of the proposed detector (Section 3). We follow with our experimental results (Section 4) and conclusions (Section 5).

\section{Related Work}

Multi-view face detection is still a popular topic, even after more than a decade of research. The ideal approach for handling multi-view face detection consists of explicitly modeling the three-dimensional structure of the face. This problem, however, is challenging in degraded viewing conditions and computationally expensive to solve in general. An alternative and, perhaps, more feasible method for handling the multi-pose detection task entails combining the outputs of multiple pose-specific classifiers. Here, we only review a subset of the multi-pose detection methods for the sake of brevity. Consult [18, 31, 32] for comprehensive surveys of the face detection literature.

The simplest approach is to decompose the face space into discrete, pose-specific areas and learn a unique classifier for each region. For example, Schneiderman and Kanade [24] modeled face and non-face appearances with parallel boosted classifiers for distinct poses, each of which relies on statistics computed over wavelet feature coefficients. Similarly, Wu et al. [30] constructed multiple posespecific nested cascades to address out-of-plane head rotation.
More sophisticated multi-view configurations model the relationships between poses. Li et al. introduced a detector pyramid [19], Jones and Viola presented a decision tree [13], and Huang et al. [10] proposed a Width-First Search (WFS) tree. These methods all decompose the range of poses over a hierarchical structure. The most advanced structure out of the aforementioned techniques, the WFS tree [10], estimates the pose of a detected face in a coarseto-fine fashion. The root node corresponds to detections across all pose angles, while each successive level of the tree makes more fine-grained pose estimates. Every parent node thus handles the full range of poses covered by its children. Finally, the leaves of the tree each address in-plane rotation for specific yaw and pitch intervals. An image region is classified as a face if it produces at least one path from the root to a leaf node. The pose label is given by the leaf node with the highest confidence.

For comparison, Zhu and Ramanan [33] proposed a deformable model for face detection, pose estimation, and landmark estimation. The model searches for mixtures of parts in images. The parts, which are represented with histogram-of-oriented-gradient descriptors, correspond to landmarks on the face and can be shared across mixtures. The mixtures consist of trees describing the relationships between the appearances of various poses. Detection is done independently for each mixture by optimizing an objective function over the configuration of the parts.

Our approach is generally much simpler than the detectors presented above. It is most similar to [24], as we rely on parallel classifiers that are constructed using boosting techniques and apply max-score fusion to combine outputs. In contrast, we have based our algorithm on the Viola-Jones framework [27]. We thus use Haar-like features that are easily computed along with pose-specific cascades of boosted classifiers that reject non-face regions quickly. We also perform score normalization to make the outputs of cascades with different structures comparable.

More importantly, we investigate the limitations of this algorithm with respect to challenges outside of pose. Our evaluation approach is similar to the method proposed by Rodriguez et al. [23], who suggested that meaningful measurements of face localization performance must focus on the ultimate application, e.g. face verification. We characterize the problem of detecting faces in the presence of pose variations, occlusion and blur, as these complications can prevent detection and subsequent analysis tasks.

\section{Detection Algorithm}

Our multi-pose algorithm employs score normalization and fusion techniques in a standard detection framework (see Figure 2). This scheme first computes an integral image [27] to prepare for classification. Faces are found by scanning an input image with a rectangular window across 


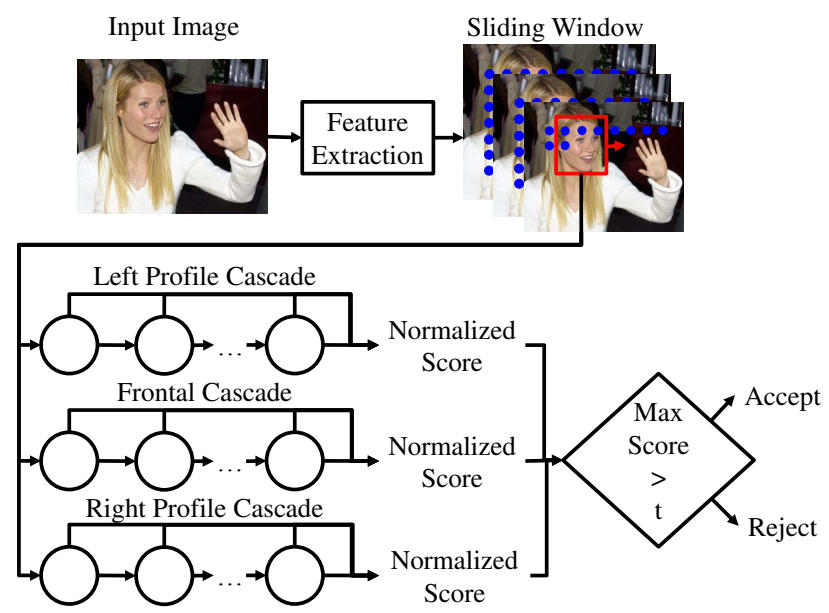

Figure 2. The proposed multi-pose detection algorithm. In the sliding window component, blue dots display prior window positions and the red rectangle covers the current position. The circles in the cascades correspond to base classifiers.

a grid pattern over a variety of scales. At each window position, the detector fuses scores from three Haar-like feature cascades [27] trained for left profile, frontal and right profile faces. Such cascades can be easily substituted with alternative classification structures. The image patch covered by the window is accepted as a face if the combined score exceeds a threshold. The windows for accepted image regions are grouped together depending on the amount of area they share in common. Each group consisting of a significant number of overlapping detections is averaged to form a single output rectangle, whereas the detections from small groups are discarded.

\subsection{Score normalization and fusion}

A Haar-like feature cascade consists of a sequence of $n$ base classifiers, $\left(c_{1}, c_{2}, \ldots, c_{n}\right)$. Each base classifier $c_{i}$ is a boosted ensemble [5] that outputs a score $s_{i}$ indicating its confidence that an image region contains a face. A classification decision is reached by comparing this score to a threshold. Any constituent classifier can reach a rejection decision for the entire cascade. In a typical cascade setup, only the $n^{\text {th }}$ classifier can indicate that an image region shows a face. If classifier $c_{i}$ with $i<n$ decides an image patch contains a face, the patch is passed along to $c_{i+1}$ for classification.

The proportion of the cascade through which an image patch passes indicates the likelihood that the patch contains a face. Similar to [11], we compute a normalized detection score based on this proportion. The normalized score accounts for both the number of cascade stages through which an image region passes as well as the score output of the last base classifier to make a decision. We first compute the minimum and maximum scores, $\min _{i}$ and $\max _{i}$, of each base classifier $c_{i}$. If $c_{i}$ belongs to the cascade for pose $p$ and outputs the final decision for a particular image patch $x$, the score for the entire cascade is given by:

$$
s_{p}(x)=\alpha \frac{i}{n}+\beta \frac{s_{i}-\min _{i}}{\max _{i}-\min _{i}},
$$

where $\alpha, \beta \in[0,1]$ are weighting parameters. We constrain the scores from each cascade to lie within $[0,1]$ by setting $\beta=1-\alpha$.

The scores of the profile and frontal cascades are fused using the max rule [15]. Let $P=\{l, f, r\}$ be the set of poses that our detector models. Then the output score for image region $x$ is

$$
s(x)=\max _{p \in P} s_{p}(x)
$$

The detector compares $s(x)$ to a threshold $t$ to classify the region.

\section{Experiments}

We evaluated the proposed detector on the FDDB data set to i) measure how well it performs on crowded scenes, and ii) characterize its failure modes. We subsequently ran this detector on pictures of the Boston Marathon crowds.

\subsection{FDDB}

The FDDB [11] contains 5170 faces pictured in 2845 challenging news article photos and internet images from the Faces in the Wild data set. The data set comprises images of faces undergoing out-of-plane rotation, significant occlusion, blur, etc. Some of the images capture densely crowded environments akin to those photographed at the Boston Marathon. Lists of manually annotated bounding ellipses serve as the ground truths for detection experiments. The authors also provide designated folds for crossvalidation, along with binaries and scripts for evaluating face detection performance.

The benchmark component of this challenge measures performance by matching the outputs of a face detector with ground truth ellipses for each image. The matching score between a detection and a ground truth region is given by the ratio of the area of intersection to the combined area of both regions. Each matched detection is assigned both a continuous score and a discrete score. The continuous score is given by the matching score. The discrete score is assigned a value of $1(0)$ if the matching score of a detection is greater (equal to or less) than one half. The true positive rate is defined either as the average continuous or discrete score for the ground truth faces. For both score types, the performance of a detector is summarized with a Receiver Operating Characteristic (ROC) curve depicting the tradeoff between the true positive rate and the number of false positives for the entire data set. 


\begin{tabular}{|lllll|}
\hline Algorithm & Features & Base classifier & Structure & Pose(s) \\
\hline Mikolajczyk et al. [22] & Orientation/blob & Boosted log-likelihood ratio & Cascade & Frontal \\
Kienzle et al. [14] & Pixels & SVM approximation & Cascade & Frontal \\
Jain et al. [12] & Haar-like & Boosted decision stumps & Adapted cascade & Frontal \\
Subburaman et al. [26] & MCT & Boosted classifiers & Guided cascade & Frontal \\
Li et al. [17] & SURF & Boosted logistic regression & Cascade & Frontal \\
Default OpenCV & Haar-like & Boosted decision stumps & Cascade & Frontal \\
FNC (this work) & Haar-like & Boosted regression stumps & Cascade & Frontal \\
PNC (this work) & Haar-like & Boosted regression stumps & Parallel cascades & Frontal/profile \\
\hline
\end{tabular}

Table 1. Face detection algorithms evaluated on the FDDB data set.

\subsection{Algorithms}

The FDDB has served as an evaluation data set for numerous algorithms, most of which have similar architectures to the Viola-Jones cascade structure. Mikolajczyk et al. [22] used local orientation and blob features in a boosted cascade to detect faces and other body parts, while Kienzle et al. [14] introduced a face detection algorithm based on an efficient approximation to the Support Vector Machine (SVM) decision function.

Later, Jain and Learned-Miller [12] extended the ViolaJones detector by reclassifying input patterns lying near the decision boundary using an online, Gaussian process regression based method. Modified Census Transform (MCT) features were used by Subburaman et al. [26] in a boosted cascade. They improved the efficiency and accuracy of the search process with an initial classification step. The best FDDB results to date for a published algorithm were obtained by Li et al. [17], who used Speeded Up Robust Features (SURF) in the boosted cascade architecture to improve performance and efficiency at training and detection time.

The implementation of the multi-pose detector described in Section 3 relies on the Viola-Jones style framework included with OpenCV 2.4.3 [1]. For the frontal face detector, we employed the classifier stored in haarcascade_frontalface_alt.xml, as Hassaballah et al. [8] found it was the best performing OpenCV detector under a wide variety of conditions. We also incorporated the cascade from haarcascade_profileface.xml to find profile faces. Features are computed twice for each image in the implementation: once for frontal and left profile faces, and another time on a reflected copy of the image for right profile faces. We refer to this implementation as the Parallel Normalized Cascades (PNC) detector.

OpenCV exposes two key parameters, including the minimum number of overlapping detection rectangles that are required for an image region to reach the output list, and the factor by which the image size is reduced at each scale. We set both of these parameters to their respective default values of 3 and 1.1. In addition, we set the score normalization weights $\alpha=\beta=0.5$ and the fused score threshold $t=0.8$. We tuned the $\alpha, \beta$ and $t$ parameters through

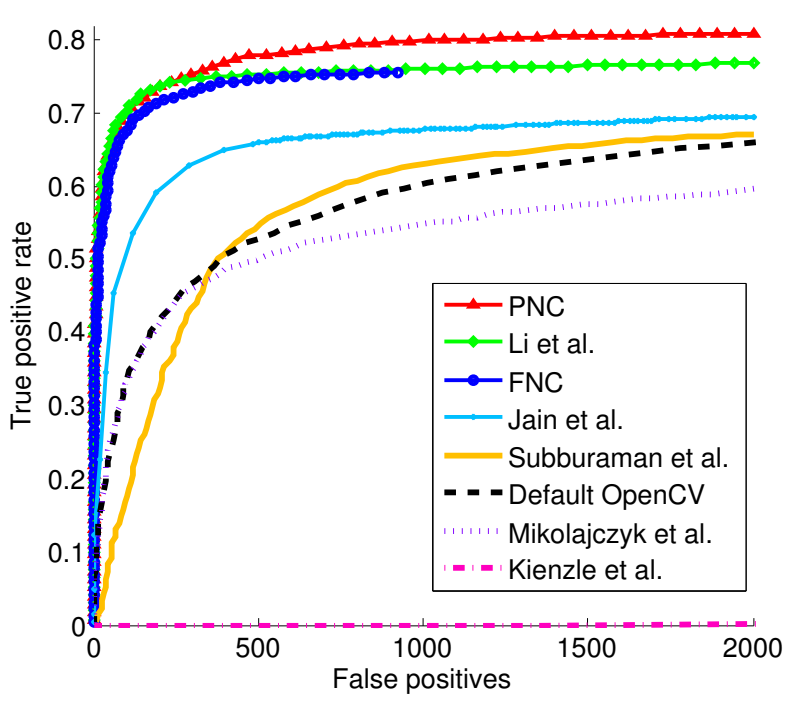

Figure 3. Discrete score ROC curve.

cross-validation using the folds provided in the FDDB. We determined that other values of $\alpha$ and $\beta$ tended to result in slightly better overall performance on the FDDB. However, we expect the naive settings stated above to generalize better across data sets. We also found that the algorithm is not particularly sensitive to the value of $t$, as values of $t \in[0.65,0.9]$ yielded similar results.

Two variations of our approach were evaluated to measure the influences of score normalization, classifier selection and pose modeling. First, the Frontal Normalized Cascade (FNC) detector is identical to the PNC scheme, except it only classifies input windows with the frontal face classifier. Second, the Default OpenCV detector matches the Viola-Jones implementation discussed in [11], so the minimum number of overlapping detections is set to 0 , the scale factor parameter is set to 1.2, and the output scores are not normalized. See Table 1 for summaries of the algorithms compared here.

\subsection{Detection performance}

The FDDB performance results show that the PNC approach generally outperformed the current state of the art 


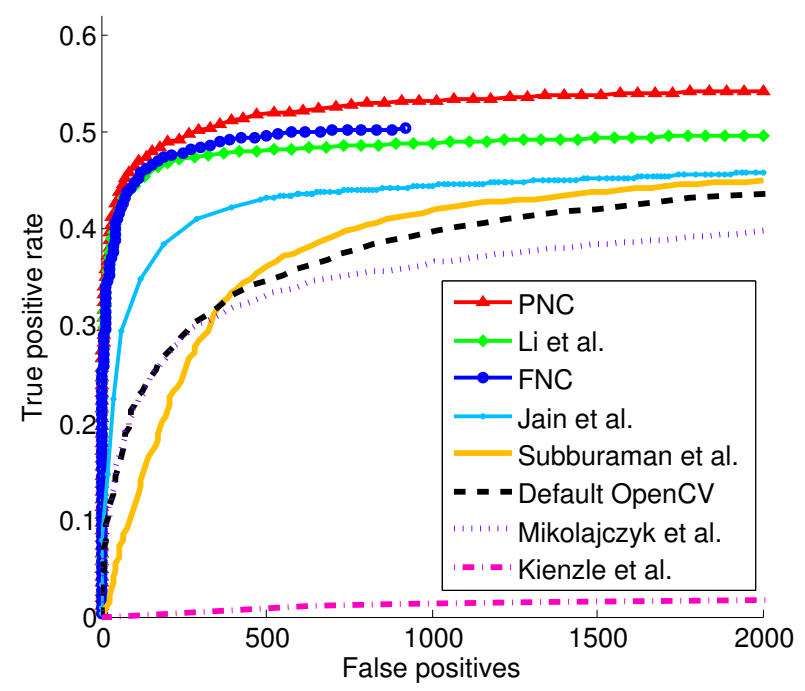

Figure 4. Continuous score ROC curve.

SURF based technique that was introduced by Li et al. [17] (see Figures 3 and 4). The true positive rates for both of these methods are comparable from 0 to 250 false positives according to the discrete score metric, at which point the PNC method begins to dominate. The performance differences are more apparent on the continuous score metric. In short, both approaches find faces equally well in some cases, but the PNC method consistently produces bounding boxes that cover the faces better than the bounding boxes of the SURF cascade.

Although the FNC algorithm performed comparably to the state of the art, the conservative default parameter values caused it to discard potentially true detections after reaching 923 false positives. Surprisingly, the improvements attained by accommodating multiple poses were negligible compared to the gains yielded by normalizing scores and using the most appropriate classifier for uncontrolled data. This tendency is demonstrated by the differences in performance between the PNC, FNC and Default OpenCV detectors.

\subsection{Effects of blur and occlusion}

Performance is still far from perfect even though we introduced measures for detecting faces with off-frontal poses. Figure 5 shows examples of detections yielded by the PNC technique. Many of the off-frontal faces were found correctly, but the missed detections were often blurry or partially out of view.

We consequently measured the impact of blur and occlusion since they both occur frequently in uncontrolled crowd scenes. We limited our analyses to the point on the ROC curve with the highest true positive rate for the PNC detector, 0.810 at 2173 false positives, because the effects of

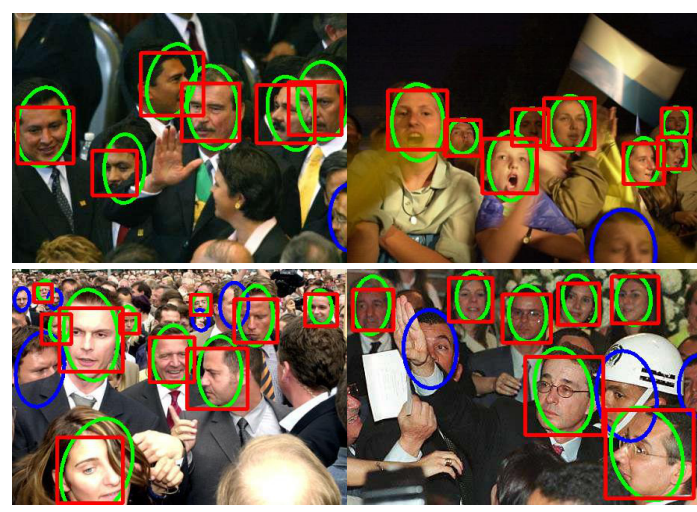

Figure 5. Examples of detections made by the PNC algorithm in crowded scenes from the FDDB data set. Green (blue) ellipses mark the ground truths for (un)detected faces, while the red squares delineate detections.

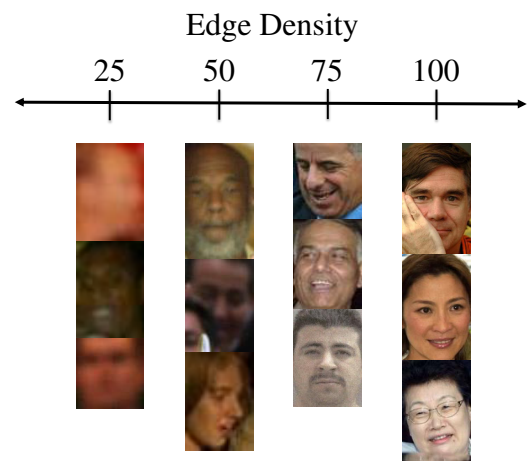

Figure 6. Reference images for a range of edge density values.

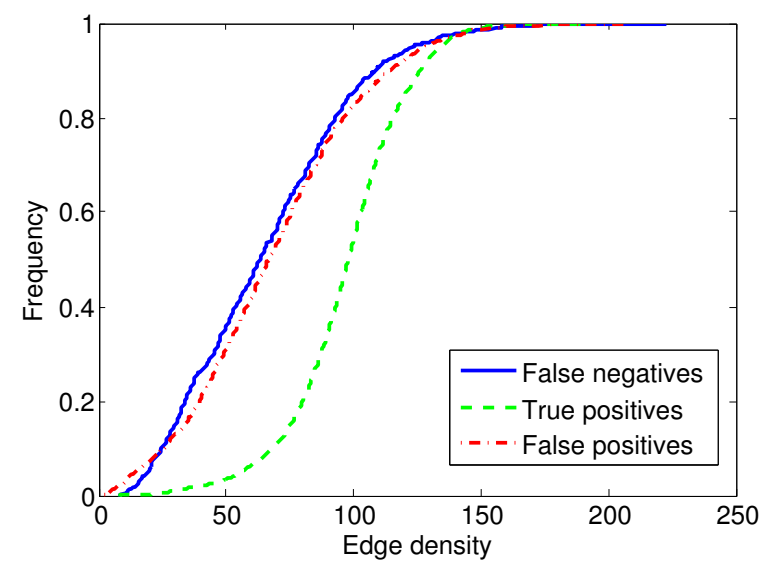

Figure 7. Empirical cumulative distribution for the edge densities of the false negatives, true positives and false positives.

blur and occlusion at this point subsume the effects at points with fewer detections. 

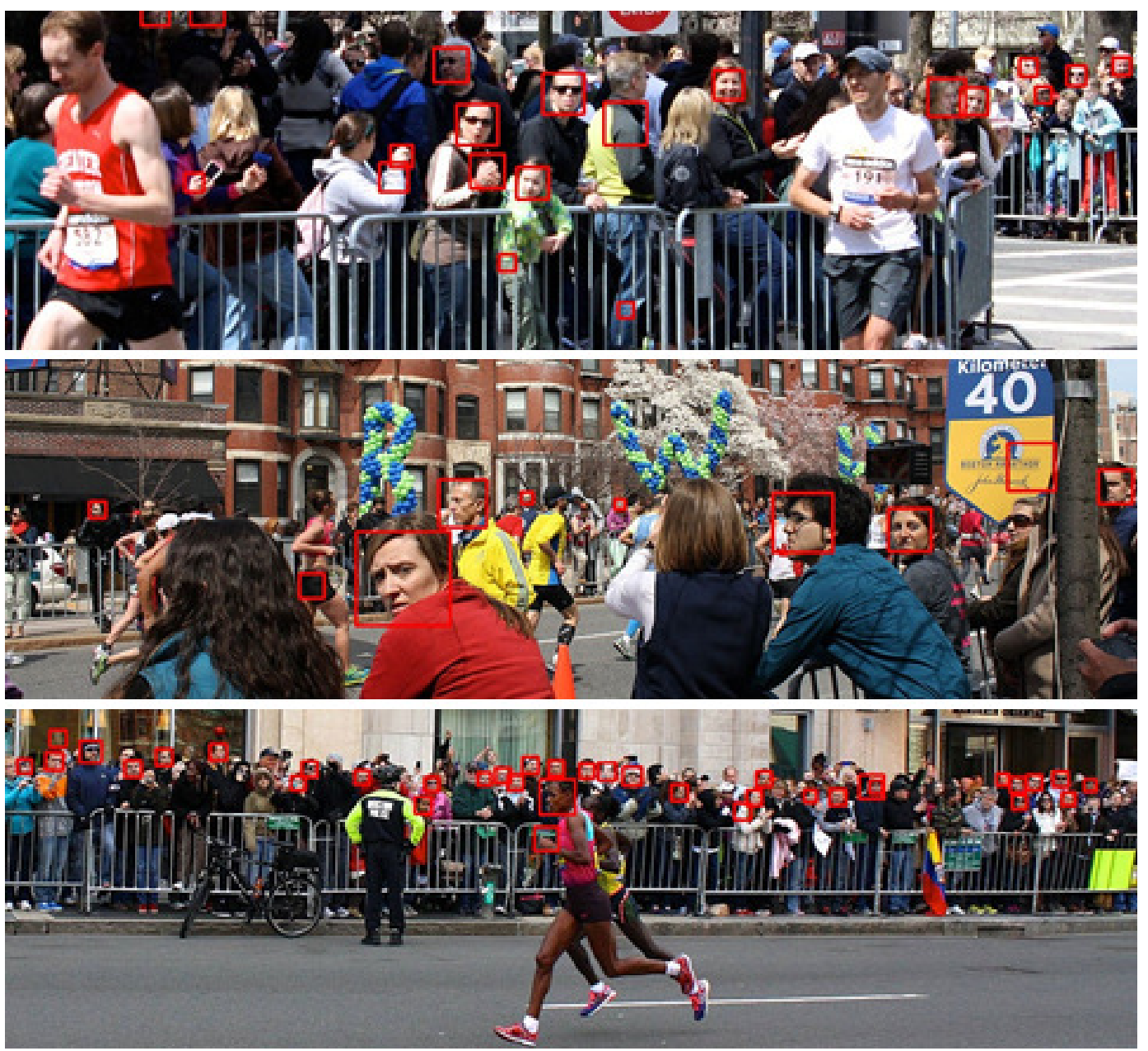

Figure 8. Examples of detections on Boston Marathon photographs. Red squares mark detections made by the PNC detector. The original, unmarked images are attributable to S. Su [25] and available under the Creative Commons Attribution 2.0 Generic license [2].

For the blur analysis, we measured blur in terms of edge density [3], which is defined as the average value of the gradient magnitude over the pixels in a region of interest. Blurry image patches tend to have lower edge density values. A relatively high value indicates than a region contains a lot of contrast changes. Sample images for a range of edge densities are shown in Figure 6. We computed the empirical cumulative distribution of the edge densities for the true positives, false negatives and false positives committed by the PNC detector on the FDDB data set (see Figure 7).

We found that $14.6 \%$ of the true positives, $62.6 \%$ of the false negatives, and $59.2 \%$ of the false positives had edge densities below 75. These differences imply that the PNC detector was able to find blurry faces in some contexts, but blurry faces were often mistaken for non-face objects and vice versa.

For the occlusion analysis, we manually labelled the true positive and false negative face images as occluded or not occluded. We applied the former label when an object blocked at least one-fifth of the facial region. Faces covered by sun-glasses, hats, hands, other crowd members, sporting equipment, etc. received the occluded label, as did faces partially lying outside of the image. Images of people with clear glasses were not assigned that label unless their faces were otherwise occluded. Likewise, faces undergoing selfocclusion due to head rotation were not treated as instances of occlusion.

The results demonstrate that occlusion contributed to a high percentage of false negatives. Approximately $48.6 \%$ of the false negatives had significant occlusion, whereas only $7.42 \%$ of the true positives were occluded. In other words, $54.2 \%$ of the occluded faces were misclassified. These results corroborate observations made by Y. Lin et al.[21], who suggested that Haar-like or similar rectangular features are sensitive to occlusion because blocked regions render the encodings unreliable. 
Our approach to mitigating out-of-plane rotation offered some performance improvements. Nevertheless, blur and occlusion effects are present in a significant number of the false negatives for the PNC detector. Further, we would expect the other algorithms that were evaluated on the FDDB to dominate the PNC and FNC detectors if they were significantly more robust to blur and occlusion. Future performance improvements will require careful attention to these nuisance factors.

\subsection{Detecting faces at the Boston Marathon}

With the aim of assessing the applicability of current face detection technology to scenarios such as the Boston Marathon incident, we ran the PNC detector on a small collection of 20 photographs. We manually tuned the scaling and quality parameters so the detector could find faces in low resolution, poor quality image patches. Figure 8 provides a sample of the results.

The PNC algorithm found some low scale faces and handled off-frontal poses well for moderate resolution faces. Conversely, it produced a significant number of false positives as a result of the manual tuning procedure, and it was not robust to simultaneous combinations of out-of-plane rotation, blur and occlusion. These limitations indicate that significant progress must be made in the area of low quality face detection before subsequent tasks, such as face recognition, can be performed on such data.

\section{Conclusions}

Multi-pose face detection has served as a popular research topic for some time [18, 32]. The gains in robustness resulting from explicitly handling just three poses are readily apparent, in light of the strong performance of the PNC algorithm relative to the frontal face methods that have been evaluated on the FDDB data set.

By comparison, face detection in the presence of occlusion and blur remains an understudied area. Representative works regarding occlusion include those of Hotta [9], who introduced an SVM face detector that used multiple kernels computed over a variety of image regions; Lin et al. [20], who proposed a multi-class variation of AdaBoost and used it to decompose the face class into region-based occlusion categories; and Goldmann et al. [6], who approached the occlusion problem by detecting four face parts independently. Blur has received even less attention from the face detection community.

Research into abating these problems in the context of higher level tasks has produced significant results. For instance, Wong et al. [29] devised a resolution detection system that facilitates the selection of the most appropriate face recognition system for comparing images with different resolutions. The lack of a sufficiently robust face detector precludes the use of such a system though. Our future work will center on improving robustness to pose, blur and occlusion using stacking [28] to integrate detectors with offsetting capabilities.

\section{References}

[1] Open Source Computer Vision. http: / / opencv . org/, retrieved May 4, 2013. 4

[2] Creative Commons Attribution 2.0 Generic. http: //creativecommons.org/licenses/by/2.0/ deed.en, retrieved May 6, 2013, April 2013. 1, 6

[3] J. Beveridge, G. Givens, P. Phillips, B. Draper, D. Bolme, and Y. Lui. FRVT 2006: Quo Vadis Face Quality. Image Vision Computing, 28(5):732-743, 2010. 6

[4] T. D. Chant. The Limits of Facial Recognition. NOVA Next, http://www.pbs.org/wgbh/nova/next/tech/ the-limits-of-facial-recognition/, retrieved May 4, 2013, April 2013. 1

[5] Y. Freund and R. Schapire. A Decision-Theoretic Generalization of On-line Learning and an Application to Boosting. Computational Learning Theory, 1995. 3

[6] L. Goldmann, U. Monich, and T. Sikora. Components and Their Topology for Robust Face Detection in the Presence of Partial Occlusions. IEEE Transactions on Information Forensics and Security, 2(3):559-569, 2007. 7

[7] J. Golen. Boston Marathon Bombing Kills 3, Injures Over 140. Associated Press, April 2013. 1

[8] M. Hassaballah, K. Murakami, and S. Ido. Face Detection Evaluation: A New Approach Based on the Golden Ratio. Signal, Image and Video Processing, 7(2):307-316, March 2013. 4

[9] K. Hotta. A Robust Face Detector under Partial Occlusion. In Proceedings of the International Conference on Image Processing, 2004. 7

[10] C. Huang, H. Ai, Y. Li, and S. Lao. High-Performance Rotation Invariant Multiview Face Detection. IEEE Transactions on Pattern Analysis and Machine Intelligence, 4(29):671686, 2007. 2

[11] V. Jain and E. Learned-Miller. FDDB: A Benchmark for Face Detection in Unconstrained Settings. Technical Report UM-CS-2010-009, Dept. of Computer Science, University of Massachusetts, Amherst, 2010. 2, 3, 4

[12] V. Jain and E. Learned-Miller. Online Domain Adaptation of a Pre-Trained Cascade of Classiers. In Proceedings of the IEEE Conference on Computer Vision and Pattern Recognition, 2011. 4

[13] M. Jones and P. Viola. Fast Multi-View Face Detection. MERL Tech Report MERLTR2003-96, 2003. 2

[14] W. Kienzle, G. Bakir, M. Franz, and B. Scholkopf. Face Detection - Efficient and Rank Deficient. Advances in Neural Information Processing Systems, (17):673-680, 2005. 4

[15] J. Kittler, M. Hatef, R. P. W. Duin, and J. Matas. On Combining Classifiers. IEEE Transactions on Pattern Analysis and Machine Intelligence, 20(3):226-239, 1998. 3

[16] J. C. Klontz and A. K. Jain. A Case Study on Unconstrained Facial Recognition Using the Boston Marathon Bombings Suspects. Michigan State University Technical Report MSUCSE-13-4, May 2013. 1 
[17] J. Li, T. Wang, and Y. Zhang. Face Detection using SURF Cascade. In Proceedings of the International Conference on Computer Vision: Workshop on Benchmarking Facial Image Analysis Technologies, 2011. 4, 5

[18] S. Li. Face Detection. In A. Jain and S. Li, editors, Handbook of Face Recognition, pages 277-303. Springer London, 2011. 1, 2, 7

[19] S. Li, L. Zhu, Z. Zhang, A. Blake, H. Zhang, and H. Shum. Statistical Learning of Multi-View Face Detection. In Proceedings of the European Conference on Computer Vision, pages $67-81,2002.2$

[20] Y. Lin and T. Liu. Robust Face Detection with Multi-Class Boosting. In Proceedings of IEEE Conference on Computer Vision and Pattern Recognition, 2005. 7

[21] Y. Lin, T. Liu, and C. Fuh. Fast Object Detection with Occlusions. In Proceedings of the European Conference on Computer Vision, pages 402-413, 2004. 6

[22] K. Mikolajczyk, C. Schmid, and A. Zisserman. Human Detection Based on a Probabilistic Assembly of Robust Part Detectors. In Proceedings of the European Conference on Computer Vision, pages 11-18, 2004. 4

[23] Y. Rodriguez, F. Cardinaux, S. Bengio, and J. Mariéthoz. Measuring the Performance of Face Localization Systems. Image and Vision Computing, 24(8):882-893, 2006. 2

[24] H. Schneiderman and T. Kanade. A Statistical Method for 3D Object Detection Applied to Faces and Cars. In Proceedings of IEEE Conference on Computer Vision and Pattern Recognition, 2000. 2

[25] S. Su. 2013 Boston Marathon. Flickr under Creative Commons Attribution, http://www.flickr.com/ photos/sonia_su/sets/72157633256126084/ with/8664753238/, retrieved May 3, 2013, April 2013. 1,6

[26] B. S. Venkatesh and S. Marcel. Fast Bounding Box Estimation based Face Detection. In Proceedings of the European Conference on Computer Vision Workshop on Face Detection, 2010. 4

[27] P. Viola and M. Jones. Rapid Object Detection using a Boosted Cascade of Simple Features. In Proceedings of the IEEE Conference on Computer Vision and Pattern Recognition, 24(1):511-518, 2001. 1, 2, 3

[28] D. Wolpert. Stacked Generalization. Neural Networks, 5:241-259, 1992. 7

[29] Y. Wong, C. Sanderson, S. Mau, and B. Lovell. Dynamic Amelioration of Resolution Mismatches for Local Feature Based Identity Inference. In Proceedings of the International Conference on Pattern Recognition, pages 1200-1203, 2010. 7

[30] B. Wu, H. Ai, C. Huang, and S. Lao. Fast Rotation Invariant Multi-View Face Detection Based on Real Adaboost. In Proceedings of the IEEE Conference on Automatic Face and Gesture Recognition, pages 79-84, 2004. 2

[31] M.-H. Yang, D. Kriegman, and N. Ahuja. Detecting Faces in Images: A Survey. IEEE Transactions on Pattern Analysis and Machine Intelligence, 1(24):34-58, 2002. 1, 2

[32] C. Zhang and Z. Zhang. A Survey of Recent Advances in Face Detection. Microsoft Research Technical Report MSRTR-2010-66, 2010. 1, 2, 7
[33] X. Zhu and D. Ramanan. Face Detection, Pose Estimation and Landmark Localization in the Wild. In Proceedings of the IEEE Conference on Computer Vision and Pattern Recognition, pages 2879-2886, 2012. 2 